\title{
CLARITY - ChiLdhood Arthritis Risk factor Identification sTudY
}

\author{
Justine A Ellis ${ }^{1,5^{*}}$, Anne-Louise Ponsonby ${ }^{2}$, Angela Pezic ${ }^{2}$, Raul A Chavez ${ }^{1}$, Roger C Allen,
} Jonathan D Akikusa ${ }^{3,4}$ and Jane E Munro ${ }^{3,4}$

\begin{abstract}
Background: The aetiology of juvenile idiopathic arthritis (JIA) is largely unknown. We have established a JIA biobank in Melbourne, Australia called CLARITY - ChiLdhood Arthritis Risk factor Identification sTudY, with the broad aim of identifying genomic and environmental disease risk factors. We present here study protocols, and a comparison of socio-demographic, pregnancy, birth and early life characteristics of cases and controls collected over the first 3 years of the study.

Methods: Cases are children aged $\leq 18$ years with a diagnosis of JIA by 16 years. Controls are healthy children aged $\leq 18$ years, born in the state of Victoria, undergoing a minor elective surgical procedure. Participant families provide clinical, epidemiological and environmental data via questionnaire, and a blood sample is collected.

Results: Clinical characteristics of cases $(n=262)$ are similar to those previously reported. Demographically, cases were from families of higher socio-economic status. After taking this into account, the residual pregnancy and perinatal profiles of cases were similar to control children. No case-control differences in breastfeeding commencement or duration were detected, nor was there evidence of increased case exposure to tobacco smoke in utero. At interview, cases were less likely to be exposed to active parental smoking, but disease-related changes to parent behaviour may partly underlie this.

Conclusions: We show that, after taking into account socio-economic status, CLARITY cases and controls are well matched on basic epidemiological characteristics. CLARITY represents a new study platform with which to generate new knowledge as to the environmental and biological risk factors for JIA.
\end{abstract}

Keywords: Juvenile idiopathic arthritis, Epidemiology, Demographics, Early life, Risk factors

\section{Background}

JIA is defined as a chronic autoimmune inflammatory arthritis of largely unknown aetiology that begins before 16 years of age [1]. It is characterised by joint swelling, pain or tenderness, and movement limitation not due to a primary mechanical disorder, that persists for at least 6 weeks. Cases are classified into seven subtypes, based on the number of joints affected and other disease features, using the International League of Associations for Rheumatology (ILAR) classification system. Current treatments are aimed at reducing pain and inflammation,

\footnotetext{
* Correspondence: justine.ellis@mcri.edu.au

${ }^{1}$ Genes, Environment and Complex Disease, Murdoch Childrens Research

Institute, Parkville, VIC, Australia

${ }^{5}$ Department of Physiology, The University of Melbourne, Parkville, VIC, Australia

Full list of author information is available at the end of the article
}

but they are largely not based on known aetiology and are thus not optimally effective [1].

The prevalence of JIA has been estimated to fall between 0.07 and 4 per thousand Caucasian children [2]. JIA can have significant impact in terms of decreased quality of life, physical function, and development [3].

JIA is typical of autoimmune disease, in that it is considered a complex disease, with susceptibility dependent on a complex interplay between inherited genetic variants [4], and life course exposure to adverse environments [5]. Amongst the relatively small number of genetic risk variants robustly identified are those in the Human Leukocyte Antigen (HLA) region, and in the shared autoimmunity gene PTPN22 [6]. Promisingly, a number of new gene loci have recently been reported, including VTCN1 [7], AFF3 [8], IL2RA [9], PTPN2 [10],

\section{Biomed Central}

(C) 2012 Ellis et al.; licensee BioMed Central Ltd. This is an Open Access article distributed under the terms of the Creative Commons Attribution License (http://creativecommons.org/licenses/by/2.0), which permits unrestricted use, distribution, and reproduction in any medium, provided the original work is properly cited. 
and C3orf1/CD80 [11] however these loci generally await further confirmation by independent studies. As we recently reviewed, less is known about the environmental factors that contribute to JIA risk [5]. Recent work identifying factors such as UVR exposure and Vitamin D, and exposure to microbes during early life (the hygiene hypothesis) and at disease onset, as important in developing other autoimmune diseases, provide hypothesis-generating clues for future research [5]. However, there is a paucity of study platforms available with which to examine environmental factors, and their interactions with gene variants.

Here, we provide detail of CLARITY, the ChiLdhood Arthritis Risk factor Identification sTudY, established in Melbourne, Australia, to address these knowledge gaps. We present study design and data collection methodologies, along with recruitment, biospecimen and environmental data collection rates, and clinical, demographic, prenatal, birth and lifestyle characteristics of the first 314 cases and 481 controls recruited to CLARITY, from study commencement in February 2008 until December 2010.

\section{Methods}

\section{Case recruitment}

All CLARITY protocols are approved by the Human Research Ethics Committee of the Royal Children's Hospital, Melbourne Australia. All participants provided written consent.

Cases are recruited during a public or private clinic visit to the Royal Children's Hospital (RCH), Victoria, Australia. The $\mathrm{RCH}$ is located in central Melbourne, and is the major paediatric tertiary referral hospital in the state. An estimated $80 \%$ of all Victorian JIA cases attend the $\mathrm{RCH}$ paediatric rheumatology clinic serviced by three rheumatologists.

Case inclusion criteria are that the child is aged between $0-18$ years at interview, with diagnosis of JIA by a paediatric rheumatologist before the age of 16 years. Exclusion criteria are the presence of major congenital abnormalities, or illness that would forgo school attendance in the one year prior to recruitment. Incident cases are defined as children recruited within six months of diagnosis. Prevalent cases are defined as those children diagnosed more than 6 months before recruitment, and since 1997. Cases were diagnosed with JIA using the ILAR criteria [12]. Case families complete a questionnaire gathering information about the child's birth; the first years of life; the household and family; skin type, sun exposure and activities; sleep habits; health problems; teeth; atopic disease; and family illness history. Additionally, case families complete a questionnaire gathering information about the parents, including socio-demographic measures, ancestry, lifestyle during the child's pregnancy (e.g. smoking, alcohol use), contact with animals and sick people, illnesses, and sun exposure; skin type; current smoking and smoking indoors near the child. Cases provide a $9 \mathrm{ml}$ peripheral blood sample.

\section{Control recruitment}

In recognition of the difficulties in obtaining a blood specimen from population/community-based child controls, controls are recruited through the Royal Children's Hospital Day Surgery Unit (RCH DSU).

Families are invited to participate if their child is aged between $0-18$, a patient of the RCH DSU for the purposes of elective surgery, and was born in the state of Victoria. This criterion allows the representativeness of the hospital based control group to the Victorian paediatric population to be eventually assessed via comparison of collected birth summary data to that collected within the Victorian Perinatal Data Collection Unit (VPDCU) [13]. Differences in demographic characteristics of controls compared to the Victorian population as a whole can then be accounted for during data analyses using back-weighting adjustments, as we have used in previous work [14]. Cases were not similarly restricted in recognition of their more limited availability, and the utility of all cases for genomic analyses regardless of place of birth. Control families are excluded if the child has major congenital abnormalities, or illness that would forgo school attendance in the year prior to recruitment. Control families complete questionnaires covering the same child and parent items as for the cases. Control children provide a $9 \mathrm{ml}$ peripheral blood sample, collected prior to anaesthesia.

\section{Questionnaire data}

In most instances, questionnaires are completed on the day of recruitment in the presence of a research nurse. Occasionally, due to time constraints, the questionnaire is completed at home. A research nurse is made available to participants by telephone to answer queries. Data is scanned directly into a comma separated file using the Teleform ${ }^{\odot}$ system.

\section{Biobanking procedures}

The peripheral blood sample is collected into EDTA and immediately delivered to the onsite biobanking facility. Plasma is removed within 2 hours and stored at $-80^{\circ} \mathrm{C}$. Within 24 hours, peripheral blood mononuclear cells (PBMCs) are isolated using a standard ficoll procedure, chilled slowly to $-80^{\circ} \mathrm{C}$, then transferred to vapourphase liquid nitrogen storage to maintain cell viability. The remaining white blood cells (mainly consisting of granulocytes) are also isolated for extraction of genomic DNA. 
Consent is also sought from both cases and controls to access Newborn Screening Cards - newborn (within 72 hours) blood spotted to card for over $99 \%$ of Victorian births for screening of treatable disease such as phenylketonuria (PKU). These cards are retained indefinitely by Genetic Health Services Victoria (housed at RCH). Newly developed methods allow the use of these blood spots for analysis of potential disease biomarkers, such as vitamin D [15], and DNA methylation [16].

\section{Statistical analyses}

In this paper we present data collected to CLARITY from February 2008 (study commencement) until December 2010, for a comparison of collected data pertaining to socio-demographics, lifestyle and birth between cases and controls. Limiting data to participants recruited by December 2010 provided a 12 month window to clarify clinical diagnosis of JIA, prior to finalisation of data for analysis.

Logistic regression was used to examine case-control differences. Analyses were carried out on the full dataset, and on the dataset restricted to cases (and controls) born in Victoria. Unadjusted odds ratios (UOR) were calculated. ORs for Victorian born cases vs controls were then adjusted (AOR) for maternal socio-economic status using SEIFA score (Socio-Economic Indexes for Areas, a ranking of geographic postal-code areas in terms of socio-economic characteristics, Australian Bureau of Statistics) [17], child age, sex and Caucasian ancestry (yes/no), and maternal age at the child's birth. Paternal SEFIA and age at child's birth were not additionally adjusted for since maternal and paternal measures were highly correlated (SEIFA score $r=0.90$, $\mathrm{p}<0.0001$; age at child's birth $\mathrm{r}=0.70, \mathrm{p}<0.0001$ ). Further adjustment for parental age at interview did not materially alter the ORs. A similar approach was taken for the comparison of older ( $>6$ years) to younger ( $\leq 6$ years) diagnosed case data, except that these analyses were carried out on all cases, and were not adjusted for child age. A p $<0.05$ was considered significant. All analyses were performed using Stata v11 (StataCorp, College Station, TX).

\section{Results}

\section{Recruitment and collection statistics}

Of the cases, 95\% (314/330) of families that were deemed eligible by our criteria took up an invitation to participate. For controls identified within the $\mathrm{RCH}$ DSU, we have achieved an overall recruitment rate of $89 \%(481 / 540)$ by face-to-face recruitment of families following child admission procedures and prior to entering theatre.

The questionnaire was completed (or partially completed) by $87 \%(272 / 314)$ of cases, and $95 \%$ (458/481) of controls. Plasma was collected for 91\% (286/314) of cases and 98\% (471/481) of controls, and PBMCs were collected for $86 \%(270 / 314)$ of cases and 90\% (433/481) of controls.

\section{Completeness of data}

Eight cases were excluded from analysis because, although their diagnosis at recruitment was JIA, this diagnosis had changed by commencement of data analysis. Data from two cases who withdrew from the study was also excluded. Examination of Table 1 demonstrates that data is not complete for all variables for the remaining 262 cases and 458 controls who completed/ partially completed questionnaires. In general, the proportion of missing data is low. However, several changes to the questionnaires occurred following study piloting. Variables added post-study piloting include SEIFA score, mode of delivery, and indoor parental smoking.

\section{Case-control data comparisons}

Table 1 displays characteristics of all cases, Victorianborn cases, and Victorian-born (all) controls. Table 2 summarises logistic regression analyses comparing Victorianborn cases and controls for selected variables, adjusting sequentially for mother's SEIFA score, child measures (age, sex, ancestry), and mother's age at the child's birth. Below, we highlight some of the key findings from data presented in these tables.

\section{Socio-demographic data}

Based on SEIFA score, mothers of Victorian-born cases were generally more advantaged than mothers of controls. Data for fathers was similar to that for mothers. More mothers of Victorian-born cases were married at the time of interview. There was evidence that mothers of cases were more highly educated, but worked fewer paid hours, than mothers of controls, following covariate adjustments.

\section{Parental lifestyle}

Fewer mothers and fathers of Victorian-born cases reported any smoking at the time of interview; this difference remained significant following regression adjustments for the mother, but not the father. The number of reported alcoholic drinks per week at the time of interview was not different between cases and controls for either mothers or fathers.

\section{Pregnancy and birth}

All data collected on pregnancy and birth pertains to the pregnancy and birth of the child recruited to the CLARITY study.

Both mothers and fathers of Victorian-born cases were significantly older at the time of the child's birth. 
Table 1 Comparison of basic data collected in CLARITY cases and controls

\begin{tabular}{|c|c|c|c|c|c|c|}
\hline & \multicolumn{2}{|r|}{ All cases } & \multicolumn{2}{|c|}{ Victorian-born cases } & \multicolumn{2}{|c|}{ Victorian-born (all) controls } \\
\hline & $\mathrm{n} \dagger$ & & $\mathrm{nt}$ & & $\mathrm{nt}$ & \\
\hline \multicolumn{7}{|l|}{ Basic characteristics } \\
\hline Child mean age at interview & 262 & 9.5 (SD: 4.5$)^{*}$ & 229 & $9.4(S D: 4.6)^{*}$ & 458 & 7.1 (SD 4.2) \\
\hline Child female & 262 & $67.2 \% *$ & 229 & $68.1 \% *$ & 458 & $39.7 \%$ \\
\hline Child has four Caucasian grandparents & 217 & $87.1 \% *$ & 190 & $90.0 \% *$ & 378 & $79.1 \%$ \\
\hline Mother's age at interview & 256 & $40.3(S D: 6.0)^{*}$ & 224 & 40.5 (SD: 5.8$)^{*}$ & 449 & 36.9 (SD: 6.4) \\
\hline Father's age at interview & 243 & $43.0(\mathrm{SD}: 6.6)^{*}$ & 213 & $43.2(6.5)^{*}$ & 398 & 39.4 (SD: 6.8) \\
\hline \multicolumn{7}{|l|}{ Socio-demographic data } \\
\hline Mother's SEIFA score & 90 & 1026.7 (SD: 62.9)* & 74 & $1030.9{\text { (SD: } 62.6)^{*}}^{2}$ & 305 & 1003.7 (SD: 70.4) \\
\hline Father's SEIFA ${ }^{\S}$ score & 81 & $1026.1(S D: 64.3)^{*}$ & 67 & $1033.6(\text { SD: } 61.7)^{*}$ & 245 & 1006.5 (SD: 71.7) \\
\hline \multicolumn{7}{|l|}{ Mother's education } \\
\hline Completed year 12 & 257 & $66.2 \%$ & 224 & $68.8 \%$ & 449 & $71.9 \%$ \\
\hline Completed postgraduate degree & 202 & $10.4 \%$ & 173 & $10.4 \%$ & 344 & $10.8 \%$ \\
\hline \multicolumn{7}{|l|}{ Father's education } \\
\hline Completed year 12 & 244 & $57.4 \%$ & 215 & $57.7 \%$ & 399 & $61.4 \%$ \\
\hline Completed postgraduate degree & 180 & $12.8 \%$ & 157 & $12.7 \%$ & 275 & $9.8 \%$ \\
\hline Mother's marital status at interview & 256 & & 223 & & 450 & \\
\hline married & & $79.3 \% *$ & & $80.7 \% *$ & & $70.9 \%$ \\
\hline divorced & & $7.0 \%$ & & $7.2 \%$ & & $6.2 \%$ \\
\hline separated & & $5.1 \%$ & & $4.9 \%$ & & $6.2 \%$ \\
\hline widowed & & $0.8 \%$ & & $0.5 \%$ & & $1.3 \%$ \\
\hline de facto & & $2.0 \%$ & & $1.8 \%$ & & $4.9 \%$ \\
\hline never married & & $5.9 \%$ & & $4.9 \%$ & & $10.2 \%$ \\
\hline Father's marital status at interview & 246 & & 216 & & 401 & \\
\hline married & & $84.2 \% *$ & & $84.3 \%^{*}$ & & $75.8 \%$ \\
\hline divorced & & $5.7 \%$ & & $5.6 \%$ & & $4.2 \%$ \\
\hline separated & & $3.7 \%$ & & $3.7 \%$ & & $5.7 \%$ \\
\hline widowed & & $0.8 \%$ & & $0.9 \%$ & & $0 \%$ \\
\hline de facto & & $1.2 \%$ & & $1.4 \%$ & & $3.7 \%$ \\
\hline never married & & $4.1 \%$ & & $3.7 \%$ & & $9.2 \%$ \\
\hline don't know & & $0.4 \%$ & & $0.5 \%$ & & $1.3 \%$ \\
\hline Mother hours in paid work (mean) & 220 & 23.0 (SD: 20.0) & 193 & 22.9 (SD: 19.9) & 340 & 20.2 (SD: 19.1) \\
\hline Father hours in paid work (mean) & 216 & 43.0 (SD: 15.1) & 190 & 43.3 (SD: 15.1) & 312 & 41.5 (SD: 16.5) \\
\hline Mother cigarettes/day & 255 & & 222 & & 451 & \\
\hline none & & $80.0 \% *$ & & $79.7 \% *$ & & $72.7 \%$ \\
\hline 1-10/day & & $9.8 \%$ & & $9.9 \%$ & & $14.9 \%$ \\
\hline 11-20/day & & $8.2 \%$ & & $8.6 \%$ & & $10.2 \%$ \\
\hline 21-40/day & & $2.0 \%$ & & $1.8 \%$ & & $2.0 \%$ \\
\hline 41+/day & & $0 \%$ & & $0 \%$ & & $0.2 \%$ \\
\hline Father cigarettes/day & 242 & & 213 & & 397 & \\
\hline none & & $78.1 \%$ & & $79.3 \% *$ & & $71.5 \%$ \\
\hline 1-10/day & & $7.9 \%$ & & $8.0 \%$ & & $10.8 \%$ \\
\hline 11-20/day & & $10.3 \%$ & & $9.4 \%$ & & $10.8 \%$ \\
\hline 21-40/day & & $2.9 \%$ & & $2.8 \%$ & & $6.3 \%$ \\
\hline 41+/day & & $0.8 \%$ & & $0.5 \%$ & & $0.5 \%$ \\
\hline
\end{tabular}


Table 1 Comparison of basic data collected in CLARITY cases and controls (Continued)

\begin{tabular}{|c|c|c|c|c|c|c|}
\hline Mother alcoholic drinks/wk mean (SD) & 249 & $2.2(S D: 3.8)$ & 216 & 2.4 (SD: 4.0) & 435 & 1.9 (SD: 3.0) \\
\hline Father alcoholic drinks/wk mean (SD) & 232 & 4.9 (SD: 6.6) & 204 & 4.8 (SD: 6.6) & 384 & 5.1 (SD: 7.9) \\
\hline \multicolumn{7}{|l|}{ Pregnancy \& Birth } \\
\hline Mother's age at child's birth (mean, yrs) & 256 & $30.2(S D: 4.9)^{*}$ & 224 & 30.6 (SD: 4.8$)^{*}$ & 449 & 29.3 (SD: 5.3) \\
\hline Father's age at child's birth (mean, yrs) & 243 & $33.0(\text { SD: } 5.7)^{*}$ & 213 & 33.4 (SD: 5.7$)^{*}$ & 398 & 32.0 (SD: 6.1) \\
\hline Planned pregnancy & 262 & $79.8 \%$ & 229 & $81.2 \%$ & 456 & $77.6 \%$ \\
\hline planned natural & & 73.7 & & $75.6 \%$ & & $72.8 \%$ \\
\hline planned assisted & & $6.1 \%$ & & $5.7 \%$ & & $4.8 \%$ \\
\hline unplanned & & $20.2 \%$ & & $18.8 \%$ & & $22.4 \%$ \\
\hline Child adopted & 262 & $0 \%$ & 229 & $0 \%$ & 455 & $0.7 \%$ \\
\hline Mother smoked during this pregnancy & 256 & & 223 & & 448 & \\
\hline Nil & & $84.0 \% *$ & & $83.9 \%^{*}$ & & $74.6 \%$ \\
\hline Less than daily & & $5.9 \%$ & & $6.3 \%$ & & $10.0 \%$ \\
\hline 1-10/day & & $6.3 \%$ & & $6.7 \%$ & & $9.4 \%$ \\
\hline 11-20/day & & $3.5 \%$ & & $3.2 \%$ & & $4.7 \%$ \\
\hline 21-40/day & & $0.4 \%$ & & $0 \%$ & & $1.3 \%$ \\
\hline 41+/day & & $0 \%$ & & & & $0 \%$ \\
\hline Father smoked during this pregnancy & 235 & & 207 & & 385 & \\
\hline Nil & & $77.0 \% *$ & & $78.3 \%^{*}$ & & $68.8 \%$ \\
\hline Less than daily & & $3.0 \%$ & & $2.9 \%$ & & $4.9 \%$ \\
\hline 1-10/day & & $5.1 \%$ & & $5.3 \%$ & & $9.6 \%$ \\
\hline 11-20/day & & $11.1 \%$ & & $10.1 \%$ & & $11.4 \%$ \\
\hline 21-40/day & & $3.4 \%$ & & $3.4 \%$ & & $4.9 \%$ \\
\hline $41+/$ day & & $0.4 \%$ & & $0 \%$ & & $0.3 \%$ \\
\hline Mother major illness during pregnancy & 261 & $26.1 \%$ & 228 & $25.0 \%$ & 452 & $23.0 \%$ \\
\hline \multicolumn{7}{|l|}{ Mother meds/supps during pregnancy } \\
\hline multivitamins & 245 & $24.9 \%$ & 215 & $25.1 \%$ & 409 & $30.8 \%$ \\
\hline folate & 246 & $61.4 \%$ & 216 & $60.7 \%$ & 409 & $64.8 \%$ \\
\hline calcium & 246 & $10.2 \%$ & 216 & $9.7 \%$ & 409 & $14.9 \%$ \\
\hline iron & 246 & $35.4 \%$ & 216 & $35.6 \%$ & 409 & $40.3 \%$ \\
\hline vitamin D & 246 & $3.3 \% *$ & 216 & $3.7 \%$ & 409 & $7.8 \%$ \\
\hline fish oil & 246 & $2.9 \%$ & 216 & $2.8 \%$ & 409 & $6.4 \%$ \\
\hline antibiotics & 246 & $3.7 \%$ & 216 & $3.7 \%$ & 409 & $3.7 \%$ \\
\hline other & 244 & $11.5 \% *$ & 214 & $11.2 \%$ & 409 & $6.4 \%$ \\
\hline Mother any alcohol during pregnancy & 255 & $18.1 \%$ & 222 & $19.4 \%^{*}$ & 448 & $18.3 \%$ \\
\hline Mother any coffee during pregnancy & 253 & $56.1 \% *$ & 220 & $57.7 \%^{*}$ & 448 & $54.7 \%$ \\
\hline Child's gestation (mean, weeks) & 211 & 39.3 (SD: 1.8) & 186 & 39.3 (SD: 1.8) & 389 & 39.0 (SD: 2.3) \\
\hline Child mode of delivery & 90 & & 71 & & 210 & \\
\hline normal vaginal & & $63.3 \%$ & & $63.4 \%$ & & $60.0 \%$ \\
\hline assisted vaginal & & $12.2 \%$ & & $12.7 \%$ & & $11.9 \%$ \\
\hline caesarian & & $23.3 \%$ & & $22.5 \%$ & & $27.1 \%$ \\
\hline other & & $1.1 \%$ & & $1.4 \%$ & & $1.0 \%$ \\
\hline Child birthweight (mean, g) & 225 & 3354.0 (SD: 613.8) & 200 & 3357.2 (SD: 581.5) & 344 & 3363.0 (SD: 676.9) \\
\hline Child birthlength (mean, $\mathrm{cm}$ ) & 194 & 50.2 (SD: 3.4) & 174 & 50.1 (SD: 3.4) & 268 & 50.4 (SD: 3.7) \\
\hline Child head circumference (mean, cm) & 161 & 34.8 (SD: 3.0) & 149 & 34.8 (SD: 3.1 ) & 176 & 34.6 (SD: 3.1$)$ \\
\hline
\end{tabular}


Table 1 Comparison of basic data collected in CLARITY cases and controls (Continued)

\begin{tabular}{|c|c|c|c|c|c|c|}
\hline Child in multiple birth & 253 & $3.6 \%$ & 221 & $4.1 \%$ & 448 & $3.1 \%$ \\
\hline Child birth order (all live-born sibs) mean & 262 & 1.90 (SD:1.02) & 229 & 1.93 (SD: 1.05) & 456 & 1.85 (SD: 1.03) \\
\hline \multicolumn{7}{|l|}{ Early Life } \\
\hline \multicolumn{7}{|l|}{ Child breastfeeding } \\
\hline any & 260 & $85.8 \%$ & 228 & $86.4 \%$ & 454 & $81.3 \%$ \\
\hline if any, age started (wks) (mean, SD) & 217 & $0.3(S D 1.6)$ & 191 & $0.3(S D 1.7)$ & 360 & 0.9 (SD 5.0) \\
\hline if any, weeks breastfed (mean, SD) & 214 & 36.5 (SD 29.9) & 188 & 36.5 (SD 30.4) & 357 & $37.6(S D$ 34.4) \\
\hline \multicolumn{7}{|l|}{ Child formula feeding } \\
\hline age started (weeks) & 194 & 18.2 (SD 19.9) & 173 & 18.3 (SD 20.1) & 348 & 16.4 (SD 16.5) \\
\hline weeks formula fed & 239 & 35.1 (SD 34.3) & 210 & $35.9(35.2)$ & 433 & $37.2(S D$ 37.1) \\
\hline Child cow's milk commence age (weeks) & 221 & 58.8 (SD 17.6) & 193 & $57.9(\mathrm{SD} 17.6)^{*}$ & 405 & $61.6(S D$ 22.4) \\
\hline Child solids commence age (weeks) & 234 & $24.2(S D$ 16.1) & 210 & 23.8 (SD 16.4) & 424 & 23.9 (SD 9.6) \\
\hline Mother smokes indoors near child & 241 & & 211 & & 216 & \\
\hline usually & & $0.8 \%$ & & $1.0 \%$ & & $1.4 \%$ \\
\hline sometimes & & $3.7 \%$ & & $3.8 \%$ & & $6.9 \%$ \\
\hline never & & $95.4 \%$ & & $95.3 \%$ & & $91.7 \%$ \\
\hline Father smokes indoors near child & 231 & & 203 & & 192 & \\
\hline usually & & $1.3 \%$ & & $1.0 \%$ & & $2.6 \%$ \\
\hline sometimes & & $3.5 \%$ & & $3.5 \%$ & & $5.7 \%$ \\
\hline never & & $95.2 \%$ & & $95.6 \%$ & & $91.7 \%$ \\
\hline
\end{tabular}

† Number of observations.

${ }^{*} p<0.05$ vs controls by unadjusted logistic regression. See Table 2 for adjusted data.

$\S$ SEIFA = Socio-Economic Indexes for Areas, a measure of residential positional disadvantage based on the Australian Bureau of Statistics Census of Population and Housing. The higher the score, the lower the disadvantage.

Significantly fewer mothers and fathers of Victorianborn cases reported any smoking during the pregnancy, this association persisted for fathers, but not for mothers, following covariate adjustments.

There was some evidence of association of JIA with nutritional supplementation during pregnancy. Use of vitamin D and fish oil during pregnancy was lower in case mothers; however, these associations were not significant for Victorian-born participants following covariate adjustments.

No differences between cases and controls were observed for gestation length or mode of delivery (including caesarean vs non-caesarean birth). Similarly, birth weight, birth length, head circumference and frequency of a child born within a multiple birth were not different between the groups. In relation to other siblings, the child's birth order was also not different between cases and controls.

\section{Early life}

Breastfeeding was commenced in $86 \%$ of Victorian-born cases, and $81 \%$ of controls; these differences were not significant. Amongst those who were breastfed, there were no significant differences between Victorian-born case and control children for breastfeeding duration. Amongst those who were formula fed, there were also no significant differences in age at commencement. A significant difference was detected for age at cow's milk introduction; on average, Victorian-born cases commenced cow's milk at a younger age than controls. However, this association did not persist following covariate adjustments. No differences in the age at commencement of solids were detected.

The frequency of both mothers and fathers who reported any smoking indoors near the child was lower in Victorian-born cases compared to controls. This difference was significant following covariate adjustments for fathers, but not for mothers. For these analyses, completion of high school by the mother was used in place of SEIFA score as a measure of socioeconomic status, since there was insufficient data for SEIFA score in the model, and the two socioeconomic covariates were significantly correlated $(\mathrm{p}<0.0001)$.

\section{Comparison of data between younger- and older- diagnosed cases}

We also considered whether differences might be evident between cases diagnosed at 6 years of age or younger (younger diagnosed cases) and cases diagnosed after 6 years of age (older diagnosed cases). We chose a 
Table 2 Unadjusted and adjusted logistic regression odds ratios (UOR/AOR) between Victorian-born cases and controls for selected phenotypic 'exposure' variables

\begin{tabular}{|c|c|c|c|c|}
\hline & \multirow[t]{2}{*}{ UOR $(95 \% \mathrm{Cl})$} & \multicolumn{3}{|c|}{ AOR $(95 \% \mathrm{Cl})$} \\
\hline & & Mother's SEIFA score & Child measures $\dagger$ & Mother's age at child's birth \\
\hline Mother's SEIFA score* & $1.01(1.00,1.01), p=0.003$ & $* *$ & $1.01(1.00,1.01), p=0.004$ & $1.01(1.00,1.01), p=0.011$ \\
\hline Mother completed Yr12 & $0.86(0.61,1.22), p=0.39$ & $1.02(0.55,1.88), p=0.95$ & $2.95(1.30,6.68), p=0.009$ & $2.72(1.19,6.20) p=0.017$ \\
\hline Father completed Yr12 & $0.86(0.61,1.20), p=0.37$ & $0.64(0.37,1.11), p=0.11$ & $1.05(0.55,1.98), p=0.89$ & $0.98(0.51,1.86), p=0.94$ \\
\hline Mother married at interview & $1.71(1.15,2.52), p=0.007$ & $1.82(0.96,3.45), p=0.065$ & $2.94(1.32,6.57), p=0.009$ & $2.62(1.16,5.92), p=0.020$ \\
\hline Father married at interview & $1.67(1.08,2.59), p=0.022$ & $2.11(1.02,4.39), p=0.044$ & $2.78(1.20,6.43), p=0.017$ & $2.48(1.06,5.80), p=0.036$ \\
\hline Mother hours in paid work ${ }^{*}$ & $1.01(1.00,1.02), p=0.12$ & $0.99(0.98,1.01), p=0.40$ & $0.98(0.96,1.00), p=0.078$ & $0.98(0.96,1.00), p=0.037$ \\
\hline Father hours in paid work* & $1.01(1.00,1.02), p=0.23$ & $1.01(0.99,1.03), p=0.50$ & $1.02(0.99,1.04), p=0.17$ & $1.02(0.99,1.04), p=0.16$ \\
\hline Mother currently smokes & $0.68(0.46,1.00), p=0.049$ & $0.45(0.22,0.92), p=0.029$ & $0.25(0.095,0.63), p=0.003$ & $0.27(0.11,0.71), p=0.008$ \\
\hline Father currently smokes & $0.65(0.44,0.97), p=0.036$ & $0.68(0.36,1.30), p=0.25$ & $0.69(0.34,1.41), p=0.31$ & $0.80(0.39,1.64), p=0.54$ \\
\hline Mother's age at child's birth* & $1.05(1.02,1.08), p=0.002$ & $1.07(1.01,1.12), p=0.016$ & $1.08(1.02,1.15), p=0.011$ & $* *$ \\
\hline Father's age at child's birth* & $1.04(1.01,1.07), p=0.009$ & $1.04(1.00,1.09), p=0.067$ & $1.06(1.01,1.11), p=0.025$ & $* *$ \\
\hline Mother smoked during child's pregnancy (any) & $0.56(0.37,0.85), p=0.007$ & $0.77(0.41,1.45), p=0.43$ & $0.52(0.24,1.12), p=0.094$ & $0.61(0.28,1.33), p=0.21$ \\
\hline Father smoked during child's pregnancy (any) & $0.61(0.41,0.91), p=0.015$ & $0.52(0.26,0.97), p=0.039$ & $0.42(0.20,0.89), p=0.024$ & $0.46(0.21,0.99), p=0.047$ \\
\hline Mother took Vitamin D during child's pregnancy & $0.45(0.21,1.00), p=0.05$ & $0.16(0.02,1.21), p=0.075$ & $0.21(0.03,1.69), p=0.14$ & $0.26(0.03,2.10), p=0.21$ \\
\hline Mother took fish oil during child's pregnancy & $0.42(0.17,1.04), p=0.06$ & $0.45(0.10,1.99), p=0.29$ & $0.71(0.15,3.36), p=0.66$ & $0.70(0.15,3.33), p=0.65$ \\
\hline Mother drank alcohol during child's pregnancy (any) & $1.07(0.71,1 / 62), p=0.74$ & $0.72(0.35,1.48), p=0.37$ & $1.07(0.50,2.35), p=0.85$ & $0.97(0.44,2.16), p=0.94$ \\
\hline Mother drank coffee during child's pregnancy (any) & $1.13(0.82,1.57), p=0.46$ & $0.85(0.51,1.42), p=0.53$ & $0.67(0.37,1.21), p=0.19$ & $0.66(0.36,1.22), p=0.19$ \\
\hline Child was breastfed (any) & $1.46(0.94,2.29), p=0.094$ & $0.88(0.44,1.73), p=0.70$ & $0.90(0.41,1.97), p=0.79$ & $0.86(0.39,1.89), p=0.71$ \\
\hline Length of breastfeeding (if any, weeks)* & $1.00(0.99,1.00), p=0.70$ & $1.00(1.00,1.01), p=0.19$ & $1.01(1.00,1.01), p=0.25$ & $1.00(0.99,1.01), p=0.72$ \\
\hline Age at commencement of cow's milk (weeks)* & $0.99(0.98,1.00), p=0.043$ & $1.00(0.99,1.01), p=0.88$ & $0.99(0.98,1.01), p=0.45$ & $0.99(0.98,1.01), p=0.30$ \\
\hline Mother smokes indoors near child (any) & $0.55(0.25,1.21), p=0.14$ & $0.49(0.21,1.10), p=0.082 \ddagger$ & $0.32(0.10,1.01), p=0.052$ & $0.33(0.10,1.06), p=0.063$ \\
\hline Father smokes indoors near child (any) & $0.51(0.22,1.18), p=0.12$ & $0.52(0.22,1.23), p=0.14 \ddagger$ & $0.22(0.07,0.67), p=0.008$ & $0.26(0.08,0.81), p=0.020$ \\
\hline
\end{tabular}

Covariates were added to the logistic regression model sequentially from left to right.

Phenotypic variables that remained significantly different between cases and controls following full covariate adjustment are highlighted in bold.

tChild measures = age, sex, Caucasian ancestry $(\mathrm{y} / \mathrm{n})$.

${ }^{*}$ coefficients reported for continuous exposures.

₹ Mother's completion of high school used in the model as a proxy for mother's SEIFA score (correlation: $r=0.24, p<0.0001$ ) due to insufficient data for SEIFA. 
cut-point of 6 years for two reasons. Firstly, the median age at diagnosis was 6.4 years, and therefore the cases were approximately evenly distributed using this cutpoint. Secondly, there is evidence to suggest that the underlying biological characteristics of JIA may be different between cases grouped in this way, even amongst cases of the same subtype $[18,19]$.

Additional file 1: Table S1 presents full data on characteristics of younger and older-diagnosed cases. Only a few statistically significant differences were identified when these case group definitions were compared. Of note, the percentage of females was higher in younger diagnosed cases; this likely reflects the fact that common subtypes such as oligoarticular JIA that are more commonly diagnosed at a younger age are also more often diagnosed in females [20]. We also noted a difference in the age of both mothers and fathers at the time of birth of the case child. Mothers of younger diagnosed cases were older $(\beta=1.1 ; 95 \%$ CI 1.0, $1.1 ; \mathrm{p}=0.015)$ as were fathers of younger diagnosed cases $(\beta=1.1 ; 95 \%$ CI 1.0 , $1.1 ; \mathrm{p}=0.012)$ at the time of the case birth. However, these associations did not persist following adjustment for SEIFA score, and child sex and ancestry. Younger diagnosed case children were also less likely to have been born as part of a multiple birth, but not significantly so following covariate adjustments. Youngerdiagnosed children were less likely to have a mother or father who smokes indoors near the child. These differences were not significant following adjustment for mother's completion of high school (as a proxy for SEIFA score), child sex, and maternal age at birth. Insufficient data was available to add ancestry to the model. No other statistically significant differences between the younger and older case groups were observed.

\section{Discussion}

The CLARITY JIA Biobank project was established in response to the paucity of data concerning both the genetic and environmental risk factors that contribute to JIA disease risk. Since its inception, the study team have achieved high recruitment rates. For cases, this demonstrates the high motivation of case families to participate in research regarding their disease. For controls in whom there is generally no such motivation to participate, we have achieved similarly high recruitment rates. Recruitment efforts have resulted in near-complete collection of questionnaire data and biospecimens (including carefully stored plasma and viable PBMCs) across all participants.

The data presented in this paper represents participants recruited between study commencement and December 2010. The 12 month lag-time prior to finalisation of data allowed for the confirmation of diagnosis of JIA. Eight cases were excluded from analysis (and two cases withdrew) during this 12 month window due to a change in diagnosis from JIA to 'other arthritis' $(n=7)$ or 'ankylosing spondylitis' ( $\mathrm{n}=1)$, and thus the imposed diagnosis window allowed a more accurate summation of data in confirmed JIA cases.

Collection of healthy paediatric control samples, especially where a blood sample is required, is a difficult task. Ideally, the control participants would be carefully sampled to accurately reflect the demographics of the entire Victorian population. However, in studies that require the collection of a biospecimen, particularly a blood sample, hospital-based controls are a necessary compromise between population-based sampling and achievement of sufficient recruitment rates. In our setting, we will have the ability to assess the robustness of full study findings to hospital control use through the collection of data on reason for minor surgery (e.g. infection related vs non-infection related admissions), and an ability to compare control demographic and birth data to that collected for all Victorian live births to the Victorian Perinatal Data Collection Unit [21].

Overall, these early case-control data comparisons demonstrate that the case and control groups are similar in many of their pregnancy, birth, and early life characteristics. Maternal SEIFA score proved to be an important covariate; with shifts in ORs and $\mathrm{p}$ values often evident upon adjustment for this variable. Some casecontrol differences were evident, even after adjustment for maternal SEIFA score, child age, sex and ancestry, and maternal age at the child's birth. These included a higher level of education, but a lower number of hours in paid work for mothers of cases, possibly reflecting an increased parental care burden on mothers of children with chronic illness. A higher number of parents of cases were married at interview. Other characteristics were not different between cases and controls following covariate adjustments.

Smoking has been identified as a strong environmental risk factor for adult rheumatoid arthritis (RA) [22]; however our data shows no increase in the risk of JIA related to tobacco smoke exposure, either in utero, or during early life. At interview, cases were less likely to be exposed to active parental smoking, but disease-related changes to parent behaviour may partly underlie this.

Three small studies were published in the mid-1990's that examined the impact of commencement and duration of breastfeeding on JIA disease risk. Each study carried significant limitations in terms of design and sample size (reviewed in [5]). However, two studies found that there was no difference in the commencement of breastfeeding [23,24], whilst one concluded that children with 'juvenile rheumatoid arthritis' were less likely to have been breastfed [25]. In relation to duration, one study found that longer duration of breastfeeding 
was protective against JIA [24], while one study found that duration was longer in children with polyarticular JIA compared to 'pauciarticular' (oligoarticular) JIA [23]. Our data showed a slightly higher rate of breastfeeding commencement in JIA children, although this difference was not significant. There were no differences in the duration of breastfeeding between cases and controls in our dataset. Overall, our data does not support a role for commencement or duration of breastfeeding in determining disease risk. There was some evidence for earlier introduction of cow's milk in cases; earlier introduction of cow's milk protein has been associated with other paediatric immune disorders [26]. However, the difference was not significant following adjustment for SEIFA score, suggesting this difference might be more related to socio-economic status.

There is a growing body of literature as to the role of vitamin $\mathrm{D}$ in autoimmune disease, including $\mathrm{RA}$ (reviewed in [5]). Interestingly, we found a trend towards a protective effect of the use of vitamin D and fish oil nutritional supplements during pregnancy, although this effect was not significant on adjustment for covariates. Additionally, the role of microbial exposures and infections in early life as potential environmental factors that protect against autoimmune disease (the hygiene hypothesis) [27] and/or act as disease triggers [28] is of interest. Caesarean delivery has been associated with increased risk of childhood immune disorders, and it has been proposed that this may be related to a lack of exposure to vaginal and intestinal flora during birth [29]. Our data shows no risk association with caesarean birth. However, a more complete examination of the role of microbial exposure on JIA risk is required to properly address such hypotheses.

\section{Conclusions}

In summary, CLARITY is an internationally unique collection of clinical and environmental epidemiological data matched with biospecimens collected from children with JIA, and from healthy control children. Recruitment is ongoing. The data presented in this paper represents a series of cases and controls collected over the first 3 years of recruitment. Cases and controls were shown to be relatively comparable in terms of pregnancy, birth, and early life characteristics, and can be assessed for source population representativeness, providing real opportunities for novel risk factor identification in this understudied but burdensome childhood disease.

\section{Additional file}

Additional file 1: Table S1. Comparison of basic data collected in CLARITY younger diagnosed and older diagnosed cases. Data not essential to the main message of the manuscript.

\section{Competing interests}

The authors declare no competing interests.

\section{Authors' contributions}

JE, JM and ALP conceived, designed and led the study. AP managed the study data and assisted JE with statistical analyses. RC assisted with the design of biobanking protocols and performed biobanking procedures. RA and JA assisted with case study design, and JM, RA and JA assisted with recruitment through their paediatric rheumatology clinics. JE wrote the manuscript, and all authors participated in drafting the manuscript to the final version.

\section{Acknowledgements}

We gratefully thank the families who kindly participated in this study. We thank Betty Lim and Sarah Macnee for their involvement in the initiation of the study and recruitment of participants, Amanda Hawker and William Siero for assistance with control recruitment and data entry, and Susan MatthyzRosa for assistance with data entry. We acknowledge support from the National Health and Medical Research Council of Australia, Arthritis Australia, Rebecca L Cooper Foundation, LEW Carty Charitable Fund, ANZ Medical Research and Technologies in Victoria Fund, Equity Trustees Lynne Quayle Charitable Trust Fund, The Australian Academy of Science, and The Victorian State Government Operational Infrastructure Support Program. JE was supported by a National Health and Medical Research Council of Australia Capacity Building Grant in Population Health, and an Australian Research Council Future Fellowship.

\section{Author details}

${ }^{1}$ Genes, Environment and Complex Disease, Murdoch Childrens Research Institute, Parkville, VIC, Australia. ${ }^{2}$ Environmental and Genetic Epidemiology Research, Murdoch Childrens Research Institute, Parkville, VIC, Australia. ${ }^{3}$ Arthritis \& Rheumatology, Murdoch Childrens Research Institute, Parkville, VIC, Australia. ${ }^{4}$ Paediatric Rheumatology Unit, Department of General Medicine, The Royal Children's Hospital, Parkville, VIC, Australia. ${ }^{5}$ Department of Physiology, The University of Melbourne, Parkville, VIC, Australia.

Received: 13 August 2012 Accepted: 8 November 2012

Published: 15 November 2012

\section{References}

1. Prakken B, Albani S, Martini A: Juvenile idiopathic arthritis. Lancet 2011, 377:2138-2149.

2. Manners PJ, Bower C: Worldwide prevalence of juvenile arthritis why does it vary so much? J Rheumatol 2002, 29:1520-1530.

3. Szer I, Kimura Y, Malleson P, Southwood T: Arthritis in children and adolescents: juvenile idiopathic arthritis. USA: Oxford University Press; 2006.

4. Glass DN, Giannini EH: Juvenile rheumatoid arthritis as a complex genetic trait. Arthritis Rheum 1999, 42:2261-2268.

5. Ellis JA, Munro JE, Ponsonby AL: Possible environmental determinants of juvenile idiopathic arthritis. Rheumatology (Oxford) 2010, 49:411-425.

6. Angeles-Han S, Prahalad S: The genetics of juvenile idiopathic arthritis: what is new in 2010? Curr Rheumatol Rep 2010, 12:87-93.

7. Hinks A, Barton A, Shephard N, Eyre S, Bowes J, Cargill M, Wang E, Ke X, Kennedy GC, John $S$, et al: Identification of a novel susceptibility locus for juvenile idiopathic arthritis by genome-wide association analysis. Arthritis Rheum 2009, 60:258-263.

8. Hinks A, Eyre S, Ke X, Barton A, Martin P, Flynn E, Packham J, Worthington J, Thomson W: Association of the AFF3 gene and IL2/IL21 gene region with juvenile idiopathic arthritis. Genes Immun 2010, 11:194-198.

9. Hinks A, Ke X, Barton A, Eyre S, Bowes J, Worthington J, Thompson SD, Langefeld CD, Glass DN, Thomson W: Association of the IL2RA/CD25 gene with juvenile idiopathic arthritis. Arthritis Rheum 2009, 60:251-257.

10. Thompson SD, Sudman M, Ramos PS, Marion MC, Ryan M, Tsoras M, Weiler T, Wagner M, Keddache M, Haas JP, et al: The susceptibility loci juvenile idiopathic arthritis shares with other autoimmune diseases extend to PTPN2, COG6, and ANGPT1. Arthritis Rheum 2010, 62:3265-3276.

11. Thompson SD, Marion MC, Sudman M, Ryan M, Tsoras M, Howard TD, Barnes MG, Ramos PS, Thomson W, Hinks A, et al: Genome-wide association analysis of juvenile idiopathic arthritis identifies a new susceptibility locus at chromosomal region 3q13. Arthritis Rheum 2012, 64:2781-2791. 
12. Petty RE, Southwood TR, Manners P, Baum J, Glass DN, Goldenberg J, He X, Maldonado-Cocco J, Orozco-Alcala J, Prieur AM, et al: International League of Associations for Rheumatology classification of juvenile idiopathic arthritis: second revision, Edmonton, 2001. J Rheumatol 2004, 31:390-392.

13. Riley $\mathrm{M}, \mathrm{Griffin} \mathrm{O}$ : Validating a statewide data collection: differences in information technology resources between hospitals. Health Inf Manag 1997, 27:67-68.

14. Dwyer T, Ponsonby AL, Ukoumunne OC, Pezic A, Venn A, Dunstan D, Barr E, Blair S, Cochrane J, Zimmet P, Shaw J: Association of change in daily step count over five years with insulin sensitivity and adiposity: population based cohort study. BMJ 2011, 342:C7249.

15. Eyles $D$, Anderson $C$, Ko $P$, Jones $A$, Thomas A, Burne T, Mortensen PB, Norgaard-Pedersen B, Hougaard DM, McGrath J: A sensitive LC/MS/MS assay of $250 \mathrm{OH}$ vitamin D3 and $25 \mathrm{OH}$ vitamin D2 in dried blood spots. Clin Chim Acta 2009, 403:145-151.

16. Wong N, Morley R, Saffery R, Craig J: Archived Guthrie blood spots as a novel source for quantitative DNA methylation analysis. Biotechniques 2008, 45:423-424. 426, 428 passim.

17. Ponsonby AL, Catto-Smith AG, Pezic A, Dupuis S, Halliday J, Cameron D, Morley R, Carlin J, Dwyer T: Association between early-life factors and risk of child-onset Crohn's disease among Victorian children born 1983-1998: a birth cohort study. Inflamm Bowel Dis 2009, 15:858-866.

18. Barnes MG, Grom AA, Thompson SD, Griffin TA, Luyrink LK, Colbert RA, Glass DN: Biologic similarities based on age at onset in oligoarticular and polyarticular subtypes of juvenile idiopathic arthritis. Arthritis Rheum 2010, 62:3249-3258.

19. Hollenbach JA, Thompson SD, Bugawan TL, Ryan M, Sudman M, Marion M, Langefeld CD, Thomson G, Erlich HA, Glass DN: Juvenile idiopathic arthritis and HLA class I and class II interactions and age-at-onset effects. Arthritis Rheum 2010, 62:1781-1791.

20. Ravelli A, Martini A: Juvenile idiopathic arthritis. Lancet 2007, 369:767-778

21. Brown SJ, Lumley JM, MCDonald EA, Krastev AH: Maternal health study: a prospective cohort study of nulliparous women recruited in early pregnancy. BMC Pregnancy Childbirth 2006, 6:12.

22. Scott DL, Wolfe F, Huizinga TW: Rheumatoid arthritis. Lancet 2010, 376:1094-1108.

23. Kasapcopur O, Tasdan Y, Apelyan M, Akkus S, Caliskan S, Sever L, Arisoy N: Does breast feeding prevent the development of juvenile rheumatoid arthritis? J Rheumatol 1998, 25:2286-2287.

24. Rosenberg AM: Evaluation of associations between breast feeding and subsequent development of juvenile rheumatoid arthritis. $J$ Rheumatol 1996, 23:1080-1082.

25. Mason T, Rabinovich CE, Fredrickson DD, Amoroso K, Reed AM, Stein LD, Kredich DW: Breast feeding and the development of juvenile rheumatoid arthritis. J Rheumatol 1995, 22:1166-1170.

26. Knip M, Virtanen SM, Seppa K, llonen J, Savilahti E, Vaarala O, Reunanen A, Teramo K, Hamalainen AM, Paronen J, et al: Dietary intervention in infancy and later signs of beta-cell autoimmunity. N Engl J Med 2010, 363:1900-1908.

27. Bach JF: Infections and autoimmune diseases. J Autoimmun 2005, 25(Suppl):74-80

28. Getts MT, Miller SD: 99th Dahlem conference on infection, inflammation and chronic inflammatory disorders: triggering of autoimmune diseases by infections. Clin Exp Immunol 2010, 160:15-21.

29. Neu J, Rushing J: Cesarean versus vaginal delivery: long-term infant outcomes and the hygiene hypothesis. Clin Perinatol 2011, 38:321-331.

doi:10.1186/1546-0096-10-37

Cite this article as: Ellis et al:: CLARITY - ChiLdhood Arthritis Risk factor Identification sTudY. Pediatric Rheumatology 2012 10:37.

\section{Submit your next manuscript to BioMed Central and take full advantage of:}

- Convenient online submission

- Thorough peer review

- No space constraints or color figure charges

- Immediate publication on acceptance

- Inclusion in PubMed, CAS, Scopus and Google Scholar

- Research which is freely available for redistribution

Submit your manuscript at www.biomedcentral.com/submit
C Biomed Central 